\title{
RAINFALL PREDICTION USING DATA MINING TECHNIQUES - A SURVEY
}

\author{
B. Kavitha Rani ${ }^{1}$ and A.Govardhan ${ }^{2}$ \\ ${ }^{1}$ Department of Computer Science \&Engineering, JITS, Karimnagar, India \\ Kavi_gdk1978@yahoo.co.in \\ ${ }^{2}$ Professor of CSE, SIT, JNTUH, Hyderabad \\ govardhan_csedyahoo.co.in
}

\begin{abstract}
Rainfall is considered as one of the major components of the hydrological process; it takes significant part in evaluating drought and flooding events. Therefore, it is important to have an accurate model for rainfall prediction. Recently, several data-driven modeling approaches have been investigated to perform such forecasting tasks as multilayer perceptron neural networks $(M L P-N N)$. In fact, the rainfall time series modeling (SARIMA) involvesimportant temporal dimensions. In order to evaluate the incomes of both models, statistical parameters were used to make the comparison between the two models. These parameters include the Root Mean Square Error RMSE, Mean Absolute Error MAE, Coefficient Of Correlation CC and BIAS. Two-Third of the data was used for training the model and One-third for testing.
\end{abstract}

\section{KEYWORDS}

SARIMA, MAE, RMSE, time series

\section{INTRODUCTION}

Characteristics and amount of rainfall are not easily known until it occurs. As rainfall plays a crucial role in evaluation and management of drought and flood events, it is very important to be able to forecast rainfall. Agriculture is the predominant occupation in India, accounting for about $52 \%$ of employment and the service sector makes up a further $34 \%$ and industrial sector around $14 \%$ [4]. Agricultural and allied sectors accounted for about $60 \%$ of the total workforce in 2009 same as in 1993-94.Most of the sub-continent depends on the rainfall for the agriculture needs. The rainfall data is available for the data mining techniques which can be useful for predicting the rainfall which can be very useful for taking decisions over crop planting in the areas.

Knowledge of seasonal rainfall, in advance, helps in proper agricultural planning. The nation is, primarily, dependent on the reasonably accurate prediction of the total amount of rainfall from the beginning of June to the end of September e.g., in case of a likely below normal rainfall situation adequate arrangements could be made for the procurement, transport, and distribution of food grains.

Data Mining aims at discovering knowledge out of data and presenting it in a form that is easily compressible to humans. It is a process that is developed to examine large amounts of data routinely collected. Data mining is most useful in an exploratory analysis scenario in which there 
are no predetermined notions about what will constitute an "interesting" outcome. Data mining is the search for new, valuable, and nontrivial information in large volumes of data. Best results are achieved by balancing the knowledge of human experts in describing problems and goals with the search capabilities of computers. In practice, the two primary goals of data mining tend to be classification and prediction. Prediction [1] [2]involves using some variables or fields in the dataset to predict unknown or future values of other variables of interest. Classification [3] refers to the task of analyzing a set of pre-classified data objects to learn a model (or a function) that can be used to classify unseen data object into one of several predefined classes. Description, on the other hand, focuses on finding patterns describing the data that can be interpreted by humans. For a given a collection of records (training set) each record contains a set of attributes, out of which one of the attribute is the class attribute or class variable. Other attributes are often called independent or predictor attributes (or variables). The set of examples used to learn the classification model is called the training data set. We need to find a model for class attribute as a function of the values of other attributes. Further previously unseen records should be assigned a class as accurately as possible. A test set is used to determine the accuracy of the model. Usually, the given data set is divided into training and test sets, training set used to build the model and test set used to validate it.

\section{LITERATURE SURVEY}

The rainfall forecasting problem has been traditionally tackled using linear techniques, such as auto regressive (AR),Autoregressive-moving-average model with exogenous inputs (ARMAX), and KalmanFilter(KF), but also using nonlinear regression [5][6]Box and Jenkins, (1970)[36]. Most of the forecasting methods consider one day ahead forecast. For the rainfall a longer term forecast such as ten days ahead or a month ahead is more of interest, though it is more difficult than the one day ahead problem. In fact, there are several considerable drawbacks to the use of $\mathrm{KF}$ in rainfall forecasting application. These include (1) the necessity of accurate stochastic modeling, which may not be possible in the case for rainfall; (2) the requirement for a priori information of the system measurement and develop covariance matrices for each new pattern, which could be challenging to accurately determine and (3) the weak observability of some of temporal pattern states that may lead to unstable estimates for the forecasted value [4].

In this context, motivation for utilizing non-linear modeling approach based on the Artificial Intelligence (AI) techniques has received considerable attention from the hydrologists in the last two decades [7]. A study was conducted on detection of nonlinear response and damage detection on signal processing, and concluded that artificial neural networks (ANN) can be used for modeling and forecasting nonlinear time series. Recently ,numerous ANN-based rainfallrunoff models have been proposed to forecast stream flow [8][9][10] and reservoir inflow. In addition, neural networks and fuzzy logic have been used as effective modeling tools in different environmental processes such as waste water treatment, water treatment and air pollution. Several water quality prediction models have been developed utilizing ANN and Adaptive neuro fuzzy inference system (ANFIS)methods[11][12][19].Rainfall-runoff models utilizing ANN model showed significant level accuracy if compared with traditional regression models, used an artificial neural networks to predict the performance of a membrane bioreactor. They were able to estimate concentrations of chemical oxygen demand, phosphate, ammonia and nitrate. Altunkaynak et al. (2005)[30] used fuzzy logic modeling to forecast dissolved oxygen concentration and compared the accuracy of fuzzylogic modeling and autoregressive integrated moving average (ARIMA)[13] models in predicting water consumption in a city. They found that relative error rates for fuzzy logic and ARIMA were 2.5 and 2.8, respectively.

An Artificial Neural Network (ANN) is a flexible mathematical structure, which is capable of identifying complex non-linear relationship between input and output data set. ANN models have 
been found useful and efficient, particularly in problems for which the characteristics of the processes are difficult to describe using physical equations[11]. Artificial neural networks (ANN) are a class of models, inspired by biological nervous systems, denoted by neurons, and working in parallel. The elements are connected by synoptic weights, which are allowed to adapt through a learning process. Now a days, neural networks are applied in hydrological [13], pattern recognition, vision, speech recognition, classification, and control systems.

Hall et al. (1993, 1999)[30] and Hsu et al. (1995) [31] have applied artificial neural network for rainfall- runoff modeling. Goswamiet al. [35] have used ANNs with three layers, namely, input layer, hidden layer and output layer for experimental forecasts of all India Summer Monsoon Rainfall. French et.al.(1992) [31] discussed on rainfall forecasting using neural networks. Here an attempt to represent the rainfall process in terms of a single-hidden layer feed forward Neural Network is made (Kulshrestha et al , 2006)[31][33].

Hung, N.Q. et.al (2009)[33], studied artificial neural networks models for rainfall forecasting. Somvanshi, V.K. et.al. (2006)[33]studied the modeling and prediction of rainfall using artificial neural networks and Box-Jenkins methodology. Other applications of ANN in hydrology are forecasting daily water demands and flow forecasting.

\section{SCOPE OF THE PROBLEM}

The following are the main objectives of the study considered in this problem.

Building of SARIMA models to forecast monthly rainfall in Coastal Andhra, Telangana and Rayalaseema regions in Andhra Pradesh state in India using Box-Jenkins methodology.

Building feed-forward neural networks models to forecast monthly rainfall in Coastal Andhra, Telangana and Rayalaseema regions in Andhra Pradesh state in India.

A comparative study is carried out to investigate the forecasting capability of feed-forward neural networks model and Box-Jenkins methods, which are among those forecasting models most successfully applied in practice. This study investigates application of neural networks models and the results of which will be compared with those obtained by Box-Jenkins method.

The FFNN model performance is evaluated using percentage better comparison with SARIMA models.

\section{DATASET}

Monthly rainfall(in MM) data for Coastal Andhra, Telangana and Rayalaseema regions in Andhra Pradesh state during the years 1871-2011 is collected from Climatology \& Hydrometeorology Division, Indian Institute of Tropical Meteorology (IITM), Pune, India. This data consists of 1680 monthly observations, in which 140 years of data during 1871-2005 is used for model fitting and remaining 6 years of data during 2006-2011 is used as out-of-sample set to measure the predictability of the selected model using mean absolute error and root mean squared error statistics.

\section{FORECASTING METHODS}

Neuron model- The multilayer perception neural network is built up of simple components. In the beginning, we will describe a single input neuron which will then be extended to multiple inputs. Next, we will stack these neurons together to produce layers [4]. Finally, the layers are cascaded together to form the network. 
Single-input neuron: A single-input neuron is shown in Figure. 1. The scalar input $p$ is multiplied by the scalar weight $W$ to form $W p$, one of the terms that is sent to the summer. The other input1, is multiplied by a bias $b$ and then passed to the summer. The summer output $\mathrm{n}$ often referred to as the net input, goes into a transfer function $\mathrm{f}$ which produces the scalar neuron output a (sometimes "activation function" is used rather than transfer function and offset rather than bias).

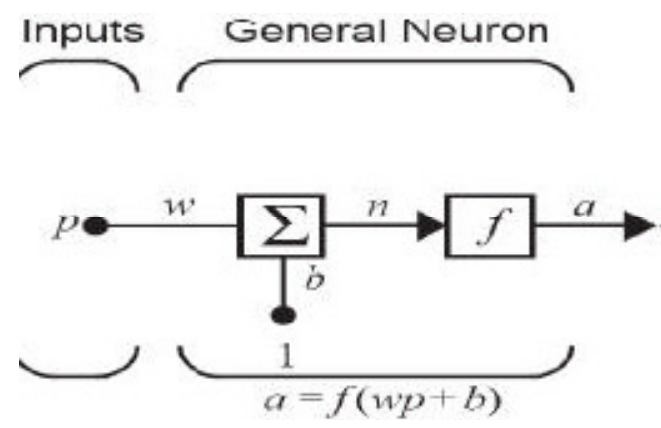

Figure 1. Single neuron

In Figure 1, both $\mathrm{w}$ and $\mathrm{b}$ are adjustable scalar parameters of the neuron. Typically the transfer function is chosen by the designer and then the parameters $\mathrm{w}$ and $\mathrm{b}$ will be adjusted by some learning rule so that the neuron input/output relationship meet some specific goal. The transfer function in Figure 1 may be a linear or nonlinear function of $\mathrm{n}$. A particular transfer function is chosen to satisfy some specification of the problem that the neuron is attempting to solve. One of the most commonly used functions is the log-sigmoid transfer function.

Multiple-input neuron: Typically, a neuron has more than one input. A neuron with $\mathrm{R}$ inputs is shown in Figure 2. The individual inputs $\mathrm{p}_{1}, \mathrm{p}_{2}, \ldots \ldots, \mathrm{p}_{\mathrm{g}}$ are each

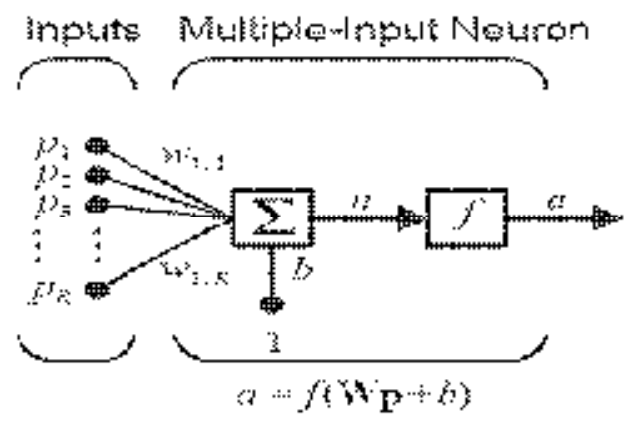

Figure 2. Multi neuron

Weighted by corresponding elements $\mathrm{W}_{1,1} \mathrm{~W}_{1,2}, \ldots \mathrm{W}_{1, \mathrm{R}}$ of the weight matrix $\mathrm{W}$. The neuron has a bias $\mathrm{b}$, which is summed with the weight inputs to form the net input $n$ :

$\mathrm{n}=\mathrm{W}_{1,1} \mathrm{P}_{1}+\mathrm{W}_{1,2} \mathrm{P}_{2}+\ldots+\mathrm{W}_{1, \mathrm{R}} \mathrm{P}_{\mathrm{R}}+\mathrm{b}$

This expression can be written in matrix form as: 
$\mathrm{n}=\mathrm{Wp}+\mathrm{b}$

Where the matrix $\mathrm{W}$ is the single neuron case has only one row. Now the neuron output can be written as:

$\mathrm{a}=\mathrm{f}(\mathrm{Wp}+\mathrm{b})(5)$

A particular convention in assigning the indices of the elements of the weight matrix has been adopted [4].The first index indicates the particular neuron destination for the weight. The second index indicates the source of the signal fed to the neuron. Thus, the indices in $\mathrm{W}_{1,2}$ say that this weight represents the connection to the first (and only) neuron from the second source [4]. A multiple-input neuron using abbreviated notation is shown in Figure 2.As shown in Figure 2, the input vector $\mathrm{p}$ is represented by the solid vertical bar at left. The dimensions of $\mathrm{p}$ are displayed below the variable as $R x l$, indicating that the input is a single vector of $\mathrm{R}$ elements. These inputs go to the weight matrix $\mathrm{W}$, which has $\mathrm{R}$ columns but only one row in this single neuron case. A constant 1 enters the neuron as input and is multiplied by a scalar bias $b$. The net input to the transfer function $f$ is $n$, which is the sum of the bias $b$ and the product $W p$. The neuron's output is a scalar in this case. If there exit more than one neuron, thenetwork output would be a vector.

\section{MUltilayer PeRCEPTRONS}

A multilayer feed forward neural network is an interconnection of perceptrons in which data and calculations flow in a single direction, from the input data to the output. The number of layers in a neural network is the number of layers of perceptrons. MLP- neural networks consist of units arranged in layers [14][24][28]. Each layer is composed of nodes and in the fully connected network considered here each node connects to every node in subsequent layers. Each MLP is composed of a minimum of three layers consisting of an input layer, one or more hidden layers and an output layer. The input layer distributes the inputs to subsequent layers. Input nodes have linear activation functions and no thresholds. Each hidden unit node and each output node have thresholds associated with them in addition to the weights. The hidden unit nodes have nonlinear activation functions and the outputs have linear activation functions. Hence, each signal feeding into a node in a subsequent layer has the original input multiplied by a weight with a threshold added and then is passed through an activation function that may be linear or nonlinear(hidden units). Neural networks allows flexibility in modeling real world complex relationships and able to estimate the posterior probability, which provides the basis for establishing classification rule and performing statistical analysis [13]

\section{Time Series forecasting}

The Box-Jenkins method [36] is one of the most widely used time series forecasting methods in practice. It is also one of the most popular models in traditional time series forecasting and is often used as a benchmark model for comparison with any other forecasting method.

It is often difficult to identify a forecasting model because the underlying laws may not be clearly understood. In addition, hydrological time series may display signs of seasonality and nonlinearity which traditional linear forecasting techniques are ill equipped to handle, often producing unsatisfactory results. Researchers confronted with problems of this nature increasingly resort to techniques that are heuristic and non-linear. Such techniques include the use of neural networks models. 
ARIMA- ARIMA and SARIMA models are extensions of ARMA class in order to include more realistic dynamics, in particular, respectively, nonstationary in mean and seasonal behaviors.

In practice, many economic time series are nonstationary in mean and they can be modeled only by removing the nonstationary source of variation. Often this is done by differencing the series. Suppose $X_{t}$ isnonstationary in mean, the idea is to build an ARMA model on the series $w_{t}$, definable as the result of the operation of differencing the series $d$ times (in general $d=1$ ): $w_{t}=$ $\Delta^{\mathrm{d}} \mathrm{X}_{\mathrm{t}}$. Hence, ARIMA models (where I stays for integrated) are the ARMA models defined on the d-th difference of the original process:

$\Phi(B) \Delta^{\mathrm{d}} \mathrm{X}_{\mathrm{t}}=\theta(B) a_{\mathrm{t}}$

Where $\Phi(B) \Delta^{\mathrm{d}}$ is called generalized autoregressive operator and $\Delta^{\mathrm{d}} \mathrm{X}_{\mathrm{t}}$ is a quantity made stationary through the differentiation and can be modeled with an ARMA.

Consider a few examples:

- $\operatorname{ARIMA}(0,1,1)$ is $\Delta^{\mathrm{d}} \mathrm{X}_{\mathrm{t}}=a_{\mathrm{t}}-\theta_{1} a_{\mathrm{t}-1} \rightarrow$ the first difference of $\mathrm{X}_{\mathrm{t}}$ is modeled as MA (1).

- $\operatorname{ARIMA}(1,1,0)$ is $\left(1-\phi_{1} B\right) \Delta \mathrm{X}_{\mathrm{t}}=a_{\mathrm{t}} \rightarrow$ the first difference of $\mathrm{X}_{\mathrm{t}}$ is modeled as AR (1).

Note that in this case:

$$
\begin{gathered}
\left(1-\phi_{1} B\right)(1-B) \mathrm{X}_{\mathrm{t}}=a_{\mathrm{t}} \\
\left(1-B-\phi_{1} B+\phi_{1} B^{2}\right) \mathrm{X}_{\mathrm{t}}=a_{\mathrm{t}} \\
{\left[1-\left(1+\phi_{1}\right) B+\phi_{1} B^{2}\right]=a_{\mathrm{t}}}
\end{gathered}
$$

The last equation shows that $\operatorname{ARIMA}(1,1,0)$ is like an $\operatorname{AR}(2)$ where $\phi_{2}=-\phi_{1}$ and $\phi_{2}+\phi_{1}=1$. This reveals that, as we knew in advance, the stationary constraint does not hold.

Seasonal ARIMA - Often time series possess a seasonal component that repeats every observations. For monthly observations $s=12$ (12 in 1 year), for quarterly observations $s=4$ ( 4 in 1 year ). In order to deal with seasonality, ARIMA processes have been generalized: SARIMA models have been formulated.

$\Phi(B) \Delta^{\mathrm{d}} \mathrm{X}_{\mathrm{t}}=\theta(B) \propto_{t}$

Where $\propto_{t}$ is such that

${ }_{s} \phi\left(B^{S}\right) \Delta_{s}^{D} \propto_{t}={ }_{s} \Theta\left(B^{S}\right) a_{\mathrm{t}}$

hence

$\phi(B)_{\mathrm{s}} \phi\left(B^{s}\right) \Delta_{s}^{D} \Delta^{\mathrm{d}} X_{\mathrm{t}}=\theta(B){ }_{\mathrm{s}} \theta\left(B^{S}\right) \propto_{t}$

and we write $\mathrm{X}_{\mathrm{t}} \sim$ ARIMA $(\mathrm{p}, \mathrm{d}, \mathrm{q}) \times(\mathrm{P}, \mathrm{D}, \mathrm{Q}){ }_{\mathrm{s}}$. The idea is that SARIMA are ARIMA $(\mathrm{p}, \mathrm{d}, \mathrm{q})$ models whose residuals $\propto_{t}$ are ARIMA ( P, D, Q ). With ARIMA ( P, D, Q ) we intend ARIMA models whose operators are defined on $B^{S}$ and successive powers.

Concepts of admissible regions SARIMA are analog to the admissible regions for ARIMA processes; they are just expressed in terms of $B^{s}$ powers.

Now, consider some examples (specialcases): 
1. $\mathrm{X}_{\mathrm{t}}=a_{\mathrm{t}}-{ }_{\mathrm{s}} \theta_{1} a_{\mathrm{t}-12}$ is ARIMA $(0,0,0) \times(0,0,1){ }_{12}$. There is only one seasonal MA component, special by $\mathrm{s}=12, \mathrm{Q}=1$. So, the ACF is characterized by finite extension and takes value only at lag $\mathrm{k}=12$. The PACF is infinite extended with exponential decay, visible at multiple of 12 lags, which is alternate or monotonic according to the sign of $\theta_{1}$.

2. $\mathrm{X}_{\mathrm{t}}={ }_{\mathrm{s}} \theta_{1} \mathrm{X}_{\mathrm{t}-12}+a_{\mathrm{t}}$ is ARIMA $(0,0,0) \times(1,0,0){ }_{12}$. The seasonal AR component is specified by $\mathrm{s}=12, \mathrm{P}=1$. So, the ACF is characterized by infinite extension. The $\mathrm{PACF}$ is with finite extended and takes value only at lag $\mathrm{k}=12$.

\section{CONCLUSION}

For rainfall prediction, artificial neural network was applied to predict the summary rainfall data in Thailand. According to the experiments, predictions of the summary rainfall data using back propagation neural network were acceptably accuracy. In the future works, some additional inputs were employed for rainfall prediction such as Sea Surface Temperature (SST) areas around Andhra Pradesh and Southern part of India.

\section{REFERENCES}

[1] Franck Le Duff, Cristian Muntean, Marc Cuggiaa, Philippe Mabob,Predicting Survival Causes After Out of Hospital Cardiac Arrest using Data Mining Method, MEDINFO 2004 M. Fieschi et al. (Eds) Amsterdam: IOS Press

[2] Gisele L. Pappa, Anthony J. Baines and Alex A. Freitas, Predicting post-synaptic activity in proteins with data mining, bioinformatics, vol 21, Pp. ii19-ii25

[3] L. S. Kumar, y. H. Lee, j. X. Yeo and j. T. Ong, tropical rain classification and estimation of rain from z-r (reflectivity-rain rate) relationships, progress in electromagnetics research b, vol. 32, $107\{127,2011\}$

[4] A. El-Shafie, A. Noureldin, M. R. Taha, and A. Hussain, Dynamic versus static neural network model for rainfall forecasting at Klang River Basin, Malaysia Hydrol. Earth Syst. Sci. Discuss., 8, 64896532, 2011,doi:10.5194/hessd-8-6489-2011 C.L. Wu, K.W. Chau, Prediction of rainfall time series using modular soft computing methods, Engineering Applications of Artificial Intelligence,2012 Elsevier Ltd. All rights .http://dx.doi.org/10.1016/j.engappai.2012.05.023

[5] S. Alvisi, G. Mascellani, M. Franchini and A. Bárdossy, Water level forecasting through fuzzy logic and artificial neural network approaches, Copernicus GmbH, 2006

[6] Yilmaz, Abdullah Gokhan; Imteaz, MonzurAlam, ,Development of a hydrologic model using artificial intelligence for Upper Euphrates Basin in Turkey

[7] Pritpal Singh, Bhogeswar Borah, Indian summer monsoon rainfall prediction using artificial neural network, springer 2013

[8] Abhishek, Kumar,, Kumar, A., Ranjan, R. ,Kumar, S,A rainfall prediction model using artificial neural network, Control and System Graduate Research Colloquium (ICSGRC), 2012 IEEE

[9] Sunyoung Lee, Sungzoon Cho, Patrick M. Wong, Rainfall Prediction Using Artificial Neural Networks , Journal of Geographic Information and Decision Analysis, vol. 2, no. 2, pp. 233 - 242, 1998

[10] VidiBhuwana, Rainfall Runoff Modeling by Using Adaptive-Network-Based Fuzzy Inference System (ANFIS) - Case Study Ciliwung River

[11] Edvinaldrian, and yudhasetiawandjamil,application of multivariate anfis for daily rainfall Prediction: influences of training data size, makara, sains, volume 12, no. 1, april 2008

[12] RiaFaulina, , Suhartono2, Hybrid ARIMA-ANFIS for Rainfall Prediction in Indonesia, International Journal of Science and Research (IJSR), India Online ISSN: 2319-7064 Volume 2 Issue 2, February 2013

[13] Guhathakurta, P (2005) "Long-range monsoon rainfall prediction of 2005 for the districts and subdivision Kerala with artificial neural network", Current Science, 90, 773-779

[14] Rajeevan, M (2001) "Prediction of Indian summer monsoon: Status, Iproblems and prospects", Current Science. 
[15] D. Mark, "Geographical information science; critical issues in an emerging cross - disciplinary Research domain”, URISA Journal,vol. 12, February 1999.

[16] Tamil Nadu Meteorology Department, Chennai.

[17] European - Commission, "Forest Fires in Europe 2007, Technicalreport, Report No. 8, 2008.

[18] L.A. Zadeh, "Fuzzy sets", Information And control vol. 8, 1965.

[19] Nguyen T. Danh, Huyah N. Phien and AshimD.Gupta 1999,"Neural network models for river flow forecasting, "Journal ofWater SA, Vol.25.

[20] S. Lee, S. Cho and P.M. Wong 1998, "Rainfall prediction usingArtificial Neural Networks," Journal of Geographic Information andDecision Analysis, Vol. 2, No. 2.

[21] Hastenrath, S(1988) "Prediction of India Monsoon Rainfall: FurtherExploration", Journal of Climate.

[22] El-Shafie A, Reda TM, Noureldin A (2007). A Neuro-Fuzzy Model forInflowForecasting of the Nile River at Aswan High Dam. WaterResour. Manag., 21(3): 533-556.

[23] French MN, Krajewski WF, Cuykendal RR (1992). Rainfall Forecastingin Space and Time Using a Neural Network. J. Hydrol. Amsterdam,137: 1-37.

[24] Halff AH, Halff HM, Azmoodeh M (1993). Predicting Runoff from RainfallUsing Neural Networks. Proc. Engrg. Hydrol. ASCE, New York, pp.760-765.

[25] Maier HR, Dandy GC (1996). The Use of Artificial Neural Networks ForThe Prediction of Water Quality Parameters. Water Resour. Res.,32(4): 1013-1022.

[26] Maria C, Valverde R, HaroldoFraga de Campos V, Nelson Jesus F(2005). Artificial Neural Network Technique For Rainfall ForecastingApplied to The Sao Paulo Region. J. Hydrol. 301:146-162.

[27] Sahai AK, Somann MK, Satyan V (2000). All India Summer MonsoonRainfall Prediction Using an Artificial Neural Netw. Clim. Dyn., 16(4):291- 302

[28] A. Altunkaynak, Z. Şen, Steady state flow with hydraulic conductivity change around large diameter wells, hydrological processes, Wiley publisher

[29] Tony Hall, Precipitation Forecasting Using A Neural Network, Weather And Forecasting Volume 14

[30] Hsu, h.-m, m.w. Moncrieff, w.-w, tung, and c. Liu, 2006a: temporal variability of warm-season precipitation over north america: statistical analysis of radar measutement. J. Atmos. Sci

[31] French, Mark N., Witold F. Krajewski, and Robert R. Cuykendall. "Rainfall forecasting in space and time using a neural network.” Journal of hydrology137.1 (1992): 1-31.

[32] Neelamshihani., K. Kumbhar, ManojKulshreshtha, Modeling of extrusion process using response surface methodology and artificial neural networks, journal of engineering science and technology, vol. 1, no. 1 (2006) 31-40

[33] N. Q. Hung, M. S. Babel, S. Weesakul, and N. K. Tripathi,An artificial neural network model for rainfall forecasting inBangkok, Thailand, Hydrol. Earth Syst. Sci., 13, 1413-1425, 2009

[34] V. K. Somvanshi, et al., "Modeling and prediction of rainfall using artificial neural network and ARIMA techniques.” J. Ind. Geophys. Union, vol. 10, no. 2, pp. 141-151, 2006

[35] B. N. Goswami1, V. Venugopal, D. Sengupta, M. S. Madhusoodanan, Prince K. Xavier, Increasing Trend of Extreme Rain Events Over India in a Warming Environment. Science 1 December 2006: Vol. 314 no. 5804 pp. 1442-1445 DOI: 10.1126/science. 1132027

[36] Box, G.E.P. and Jenkins, G.M. (1970), Time Series Analysis: Forecasting and Control, San Francisco:Holden-Da y. 